\title{
Polyarthritis as the Initial Presentation of Hyper Eosinophilic Syndrome
}

\author{
Lubna Nazir, Amir Riaz and Hamza Alam
}

\begin{abstract}
Hyper eosinophilic syndrome (HES) is a rare condition with a potential for morbidity and mortality, if left untreated. Therefore, it is important to highlight it, as often these cases are misdiagnosed and mismanaged, specially when presenting with an atypical initial presentation. This case report describes an unusual initial clinical presentation of HES. Patient was a 75-year lady presenting to Rheumatology Clinic with short duration of fever and polyarthritis. Joint aspiration showed purulent fluid with a cell count of $61,000 / \mathrm{mm}^{3}$ with predominant neutrophils and eosinophils. Her peripheral blood also showed a high white blood cell (WBC) count $\left(80,700 / \mathrm{mm}^{3}\right.$ with $73 \%$ eosinophils). Her workup for eosinophilic leukemia was negative, so a diagnosis of HES was made. She was initiated on corticosteroids and hydroxycarbamide as first-line therapy. Unfortunately, the patient was unresponsive to steroids with her WBC count rising to $130,000 / \mathrm{mm}^{3}$ and her clinical course was complicated by cardiac failure and peripheral neuropathy. Improvement in arthritis and peripheral eosinophilia was noted after she was given imatinib and stabilised clinically.
\end{abstract}

Key Words: Hypereosinophilic syndrome, Polyarthritis, lamtinib.

\section{INTRODUCTION}

Hypereosinophilic syndrome (HES) is a myeloproliferative disorder characterised by persistent eosinophilia (absolute eosinophilic count $>1,500 / \mathrm{mm}^{3}$ ) that is associated with damage to multiple organs. ${ }^{1}$ The differential diagnosis of eosinophilia includes parasitic infections, systemic vasculitides, eosinophilic arthritis, and myopathies, together with the idiopathic HES and malignancy. HES is diagnosed when no underlying cause is recognised for chronic eosinophilia and tissue damage. The condition was first described in 1968. 2 The diagnosis of HES is based on the following criteria: persistent eosinophilia, as defined by blood counts showing increased numbers of eosinophils (greater than 1,500 eosinophils $/ \mathrm{mm}^{3}$ ) for at least 6 months, signs and symptoms of organ involvement, and no other cause of eosinophilia. ${ }^{3}$ The mortality can be as high as $75 \%$ after diagnosis in untreated patients for upto 3 years; and cardiac involvement is the most common cause of increased morbidity and mortality. ${ }^{4}$ Reports on initial rheumatologic manifestations in patients with HES are very rare.5,6

Here, we present an unusual case of purulent polyarthritis as the first presentation of HES.

\section{CASE REPORT}

A 72-year lady visited Rheumatology Clinic in November 2014 with complaints of fever and dry cough for around

Department of Rheumatology, Liaquat National Hospital,

Karachi, Pakistan

Correspondence: Dr. Hamza Alam, Department of

Rheumatology, Liaquat National Hospital, Karachi, Pakistan

E-mail: drhamza84@gmail.com

Received: November 10, 2018; Accepted: January 31, 2019
1 month and polyarthritis for preceding 4 days. Fever was initially low grade and intermittent, associated with mild dry cough but for past 4 days it had become high grade reaching up to $105^{\circ} \mathrm{F}$. It was associated with polyarthritis of both small and large peripheral joints. Except for daily paracetamol, she denied any other drug intake. She had no personal or family history of asthma, atopy, or serious illnesses. Her physical examination revealed that she was febrile $\left(103^{\circ} \mathrm{F}\right)$. She had a few small anterior cervical lymph nodes and a dry, coated tongue as well as swollen, tender and warm joints with involvement of proximal interphalangeal (PIPs), metacarpophalangeal (MCPs), wrists, elbows, shoulders, knees and ankles. There were no subcutaneous nodules. On auscultation, coarse crackles were audible on basal area of the left side of her chest. Rest of the systemic examinations were unremarkable. Synovial fluid aspiration was done in clinic and approximately 50 $\mathrm{ml}$ of frank purulent fluid was aspirated from each knee joint which was sent for synovial fluid detailed report (DR) and culture.

The patient was admitted, and in view of polyarticular septic arthritis, was started empirically on broad spectrum antibiotics. Her laboratory in investigations revealed normal hemoglobin and platelet counts, but white blood cell (WBC) count of $81,700 / \mathrm{mm}^{3}$, with $73 \%$ eosinophils seen on peripheral smear (Figure 1). Her erythrocyte sedimentation rate (ESR) was $38 \mathrm{~mm} / \mathrm{h}$ and C-reactive protein $(\mathrm{CRP})$ was 10 (normal $<0.1)$. Her serum biochemistry and liver functions were normal. Synovial fluid white cell count was $61,000 / \mathrm{mm}^{3}$ with predominant neutrophils $(75 \%)$ and eosinophils $(25 \%)$. Her antinuclear anti-body (ANA) was +1 homogenous, but anti-dsDNA, ENA profile, anti-CCP, c-ANCA and p-ANCA levels were normal. Chest $\mathrm{X}$-ray showed left 


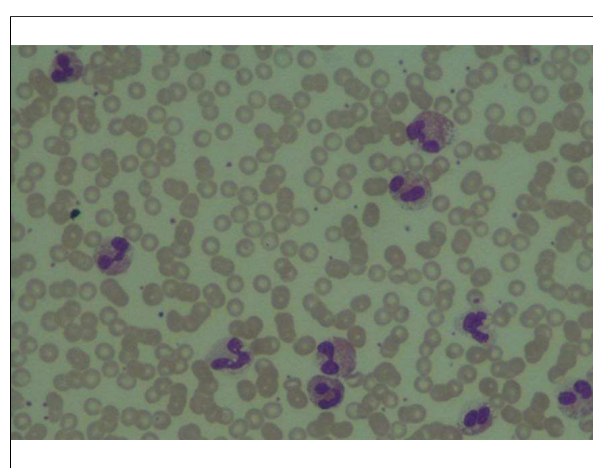

Figure 1: Photomicrograph of peripheral smea (Leishmann Stain x 40). Many bilobed eosinophils with prominent acidophilic cytoplasmic granules.

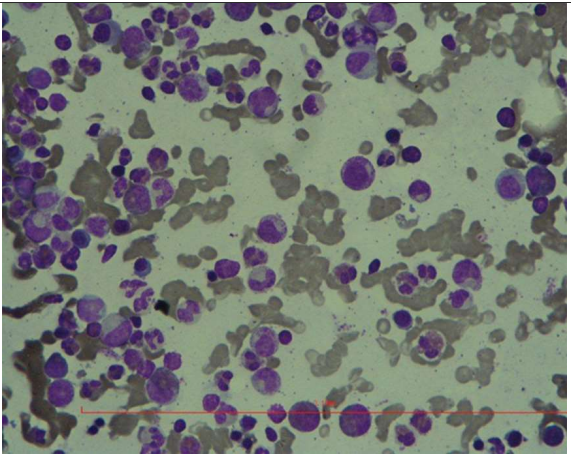

Figure 2: Photomicrograph of bone marrow aspiration biopsy (Leishmann Stain $x$ 40). Eosinophils and their precursor cells at various stages of differentiation are seen.

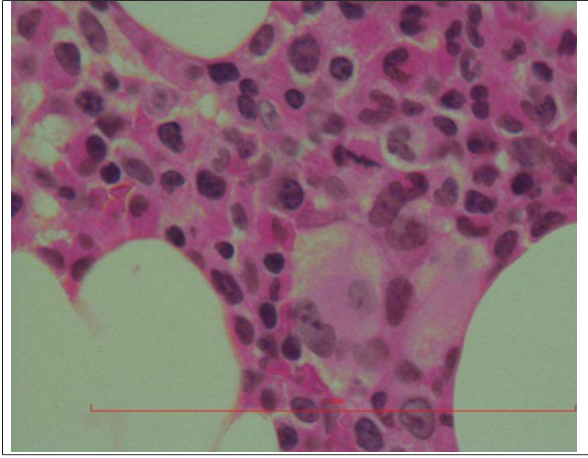

Figure 3: Photomicrograph of bone marrow trephine biopsy (H\&E Stain $\times 100$ ).

Numerous eosinophil precursor cells seen. lower zone consolidation. X-ray of her hands showed soft tissue swelling at PIPS, MCPs and wrist joints, bilaterally. Repeated blood cultures showed no growth. Patient was started empirically on antibiotics; however, her fever or her WBC count did not subside in over 48 hours. Her WBC count rose to $94,000 / \mathrm{mm}^{3}$ on day 2 and $110,000 / \mathrm{mm}^{3}$ on day 3 with $86 \%$ eosinophils. Hematology consult was given to rule out eosinophilic leukemia. Her bone marrow biopsy was done on day 3. On day 4, she developed cardiac failure and was shifted to intensive care unit (ICU). Echo showed severe global hyperkinesia with moderate mitral regurgitation and tricuspid regurgitation. Her B-natriuretic peptide was elevated $(24,690 \mathrm{pg} / \mathrm{mL})$. She developed fast atrial fibrillation which responded to amiodarone. Diuretics and anticoagulation were started. The patient was started on pulse steroid therapy, which also showed no response as she still had persistent fever; while on the other hand, peripheral WBC and eosinophilic count kept on rising to $130,000 / \mathrm{mm}^{3}$ and $83 \%$, respectively. Her bone marrow morphology, immunohistochemistry IgG, IgM and cytogenetics were negative for leukemia or lymphoma; however, it showed abundance of eosinophils and its precursors (Figures 2 and 3). Platelet-derived growth factor receptor (PGDFR) and BCR/ABL mutations were absent. CT neck, chest and abdomen showed multiple enlarged lymph nodes in neck, mediastinum, peri-hilar, peri-aortic and inguinal region. Lymph node biopsy showed reactive changes only. After ruling out other potential causes, a final diagnosis of HES was made and the patient was started on imatinib, $100 \mathrm{mg} / \mathrm{day}$ and the hydoxyurea, $500 \mathrm{mg}$ twice daily, along with oral prednisolone $1 \mathrm{mg} / \mathrm{kg} / \mathrm{day}$. Later, due to absence of PDGFR mutation, dose of imatinib was increased to $200 \mathrm{mg} /$ day. Patient then developed lower limb weakness; and nerve conduction studies showed diffuse sensory motor polyneuropathy, which was treated conservatively with physiotherapy. Within 72 hours of imatinib, her leukocyte count started to decline and her arthritis settled slowly. She was discharged on day 34 . By that time, she was moving with support and her WBC was $14,000 / \mathrm{mm}^{3}$. She visited 2 weeks after being discharged, feeling relatively well with a WBC of $9,200 / \mathrm{mm}^{3}$. Thus imatinib dose was reduced to 100 $\mathrm{mg} /$ day. Her hydroxyurea was stopped after one month but her imatinib was continued.

\section{DISCUSSION}

HES is a rare disease with heterogeneous symptoms. This case is unique as literature search does not reveal any previous such cases which presented with sterile purulent polyarthritis as initial symptoms of HES. This patient did not respond to high dose steroids but her arthritis settled after lowering of WBC count by imatinib. Joint involvement is rare in HES. Martin-Santos et al. reported a non-erosive arthropathy with tenosynovitis. ${ }^{5}$ Tay $\mathrm{CH}$ also reported 10 cases with eosinophilic large joint arthritis of unknown etiology but none had HES. ${ }^{6}$

Risk of infection is not high in HES, which explains the culture negative purulent synovitis in this patient; and the high synovial fluid white cells with elevated eosinophils could merely be a representation of a massively high blood leucocytosis and eosinophilia extravasating into the synovial fluid. Synovial fluid eosinophilia has also been reported in the past.2,5

Virtually, any organ system may be involved in HES, but the heart, central nervous system, skin, and respiratory tract are commonly involved. Cardiovascular complications of HES are a major source of morbidity and mortality with reported frequency of $40-50 \%$. The most common complications are endomyocardial fibrosis, congestive cardiac failure and mitral valve regurgitation. Myocardial infarction is rarely reported. ${ }^{7}$

Central nervous system involvement is seen with peripheral neuropathies, hemiplegia, paraplegia encephalopathy, memory loss and ataxia. ${ }^{4}$ Peripheral neuropathies present as symmetric or asymmetric sensory changes, pure motor deficits, or mixed sensory and motor complaints. ${ }^{8}$

ANA may be positive, however, it does not represent an autoimmune disorder. ${ }^{4}$ Recent advances in underlying pathogenesis have established that hypereosinophilia 
may be due to either primitive involvement of myeloid cells, essentially due to occurrence of an interstitial chromosomal deletion on $4 q 12$ leading to creation of the FIP1L1-PDGFRA fusion gene (F/P+ variant), or to increased interleukin (IL)-5 production by a clonally expanded $\mathrm{T}$ cell population (lymphocytic variant), most frequently characterised by a CD3- CD4+ pheno-type. 9

Steroid and imatinib are first line treatment options. It is unclear if imatinib can cure the disease, as cases of recurrence are reported few months after stoppage of treatment. 10 Other therapeutic options are hydroxy-carbamide, IFN- $\alpha$, monoclonal anti-IL-5 antibodies, ciclosporin, vincristine, and anti-CD52 antibodies (alemtuzumab). Treatment of cardiac damage due to the eosinophilia has ranged from intensive steroid treatment to heart transplantation. ${ }^{11}$

Prognosis depends on two major aspects of disease: heart involvement and the increased likelihood of developing hematological malignancies.

The patient described here is notable because of her unusual initial presentation with purulent polyarthritis and fulminant course with severe cardiac involvement and subsequent complications, without evidence of clonality or neoplasia. Despite a good response to therapy, long-term surveillance for hematological malignancies is mandated.

\section{REFERENCES}

1. Seifert M, Gerth J, Gajda M, Pester F, Pfeifer R, Wolf G. Eosinophilia - A challenging differential diagnosis. Med Klin (Munich) 2008; 103:591-7.

2. Hardy WR, Anderson RE. The hypereosinophilic syndrome. Ann Intern Med 1968; 68:1220-9.

3. Noh HR, Magpantay GG. Hypereosinophilic syndrome. Allergy Asthma Proc 2017; 38:78-81.

4. Venkatesh C, Mahender E, Janani S, Malathi S, Vijayakumar M, Nammalwar BR. Hypereosinophilic syndrome. Indian J Pediatr 2006; 73:237-9.

5. Martín-Santos JM, Mulero J, Andréu JL, de Villa LF, Bernaldode Quirós L, Noguera E. Arthritis in idiopathic hyper-eosinophilic syndrome. Arthritis Rheum 1988; 31:120-5.

6. Tay CH. Eosinophilic arthritis. Rheumatol 1999; 38:1188-94.

7. Parrillo JE, Borer JS, Henry WL, Wolff SM, Fauci AS. The cardiovascular manifestations of the hypereosinophilic syndrome. Prospective study of 26 patients, with review of the literature. Am J Med 1979; 67:572-82.

8. Ogbogu P, Rosing DR, Horne MK. Cardiovascular manifestations of hypereosinophilic syndromes. Immunol Allergy Clin North Am 2007; 27:457-75.

9. Chusid MJ, Dale DC, West BC, Wolff SM. The hypereosinophilic syndrome: Analysis of fourteen cases with review of the literature. Medicine (Baltimore) 1975; 54:1-27.

10. Roufosse FE, Goldman M, Cogan E. Hypereosinophilic syndrome. Orphanet J Rare Dis 2007; 2:37.

11. Brito-Babapulle $F$. The eosinophilias, including the idiopathic hypereosinophilic syndrome. Br J Haematol 2003; 121:203-23. 